\title{
A!
}

This is an electronic reprint of the original article.

This reprint may differ from the original in pagination and typographic detail.

Kupiainen, Antti; Muratore-Ginanneschi, Paolo; Pekola, Jukka; Schwieger, Kay

\section{Fluctuation relation for qubit calorimetry}

Published in:

Physical Review E

DOI:

10.1103/PhysRevE.94.062127

Published: 20/12/2016

Document Version

Publisher's PDF, also known as Version of record

Please cite the original version:

Kupiainen, A., Muratore-Ginanneschi, P., Pekola, J., \& Schwieger, K. (2016). Fluctuation relation for qubit calorimetry. Physical Review E, 94(6), 1-6. [062127]. https://doi.org/10.1103/PhysRevE.94.062127

This material is protected by copyright and other intellectual property rights, and duplication or sale of all or part of any of the repository collections is not permitted, except that material may be duplicated by you for your research use or educational purposes in electronic or print form. You must obtain permission for any other use. Electronic or print copies may not be offered, whether for sale or otherwise to anyone who is not an authorised user. 


\title{
Fluctuation relation for qubit calorimetry
}

\author{
Antti Kupiainen, ${ }^{1, *}$ Paolo Muratore-Ginanneschi, ${ }^{1, \dagger}$ Jukka Pekola, ${ }^{2, \ddagger}$ and Kay Schwieger ${ }^{1, \S}$ \\ ${ }^{1}$ Department of Mathematics and Statistics, University of Helsinki, P.O. Box 68, 00014 Helsinki, Finland \\ ${ }^{2}$ School of Science, Aalto University, P.O. Box 13500, 00076 Aalto, Finland
}

(Received 10 June 2016; published 20 December 2016)

\begin{abstract}
Motivated by proposed thermometry measurement on an open quantum system, we present a simple model of an externally driven qubit interacting with a finite-sized fermion environment acting as a calorimeter. The derived dynamics is governed by a stochastic Schrödinger equation coupled to the temperature change of the calorimeter. We prove a fluctuation relation and deduce from it a notion of entropy production. Finally, we discuss the first and second law associated with the dynamics.
\end{abstract}

DOI: 10.1103/PhysRevE.94.062127

\section{INTRODUCTION}

Speculations with regard to the fundamental bounds imposed by physical laws on information processing and transfer [1-4] has led to the following oft-raised question: How do the thermodynamic laws of the macroscopic world transduce and impinge on the behavior of nanolevel and quantum-level structures?

Developments over the past decade have demonstrated the experimental feasibility of temperature measurements in nanoscale systems with sub-microsecond time resolution. For example, high-sensitivity temperature measurements have been accomplished by embedding a superconductor-insulatornormal metal (SIN) tunnel junction into a radiofrequency (RF) resonant circuit. The temperature of the normal metal side of an SIN tunnel junction is then read by measuring the reflection or transmission coefficient of the $L C$ resonator as a function of both the temperature and an external current bias [5] (see [6] and the review [7]). SIN tunnel junction thermometers can be employed as fast calorimeters to perform basic studies of the thermodynamics of a mesoscopic nanostructure. As a consequence, questions that were restricted to speculation only a couple of decades ago are now becoming feasible in properly designed experiments.

The calorimetric measurement of the full distribution of the work done by exerting an external drive on a quantum two-level system (qubit) was proposed recently [8]. Let us recall briefly the setup that makes the proposed experiment realistically possible. The qubit is implemented using a solid-state electronic circuit [9]. A basic example is a superconducting Cooper pair box (CPB) [9-12] or a transmon qubit $[13,14]$. A CPB consists of a superconducting electrode (island) put in contact with a superconducting reservoir. Actual implementations use, e.g., a superconductor-insulator-superconductor (SIS), aluminum-aluminum oxide-aluminum $\left(\mathrm{Al} / \mathrm{Al}_{2} \mathrm{O}_{3} / \mathrm{Al}\right)$, or a tunnel junction with capacitance $C_{j}$. Charges are driven from the reservoir to the island by a voltage source $V$ between the reservoir and a capacitor $C_{g}$ connected to the island. If the energy of thermal fluctuations $k_{\mathrm{B}} T$ and the charging

\footnotetext{
*antti.kupiainen@helsinki.fi

†paolo.muratore-ginanneschi@helsinki.fi

${ }_{\ddagger}^{\ddagger}$ jukka.pekola@aalto.fi

§kay.schwieger@gmail.com
}

(Coulomb) energy of the island are much smaller than the superconducting gap $\Delta$ (which is about $1 \mathrm{~K}$ for aluminum), all electrons in the island are paired. The experiment thus needs to be performed at temperatures of the order of $0.1 \mathrm{~K}$ or below. The effective Coulomb energy of the island becomes $E_{C}=(2 e)^{2} / 2\left(C_{g}+C_{j}\right)$. Finally, tuning the circuit parameters so that $E_{C} \gg k_{\mathrm{B}} T$ ensures that only the ground state (no pair in the island) and the first excited state (one pair in the island) have non-negligible probability. A qubit dynamics is thus effectively realized ([15,16]; see also [17] and references therein).

A basic calorimeter is formed out of a normal metal island with typically $10^{9}$ electrons on it. It is only weakly coupled to the surrounding thermal bath (phonons) at these low temperatures. Calorimetric measurements of the solid-state qubit just described can then be performed by connecting the normal metal electrode as a SIN junction thermometer $[6,8]$ to the resonant circuit. The experimental setup of [8] envisages a drive signal with equal free energy at the end of the control horizon. Energy conservation then implies that up to boundary terms, the work $W$ done on the system is equal to the heat $Q$ dissipated to the environment in the time interval from the beginning of the driving until the end of the equilibration period.

In classical nonequilibrium thermodynamics, a group of relations commonly referred to as fluctuation theorems ([18-23]; see also [24-26] for a review) links the distribution of the work done on a small system during a transition from an equilibrium state to the free energy of the system at the transition end states. Classically, the work is a random functional of the protocol, i.e., the sequence of nonconservative forces exerted on the system to drive the transition. From the mathematical slant, fluctuation relations are statements about deviations in the distribution of work around zero. They are therefore obtained by comparing the work distribution during the transition and a second one specified by an appropriately defined time-reversed protocol [25].

The extension of fluctuation theorems to the quantum case poses the problem of how to define the work as a functional of the path that the system has followed during the transition. Intense research activity was recently focused on determining work for closed and open quantum systems (see [27-36]). For this reason, the experiment proposed in [8] is of interest. It calls for a detailed analysis of how fluctuation relations can be derived and used to achieve a consistent nonequilibrium 


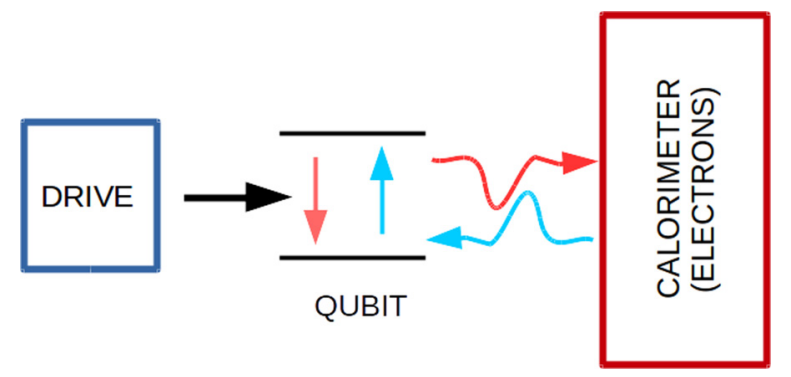

FIG. 1. Pictorial representation of the theoretical model described in Sec. II.

thermodynamic description of a driven qubit. This is the scope of the present work.

The paper contains two main results. First, a detailed theoretical model is presented for the driven qubit-calorimeter dynamics of [37] (see Fig. 1). In particular, in Sec. II we derive the stochastic Schrödinger equation (cf. $[38,39]$ ) describing the evolution of the quantum trajectories followed by a quantum system continuously monitored by an environment, in our case embodied by the calorimeter. In the experimental setup of [37], temperature measurements are possible because the calorimeter can be thought of as a fermion system with a number of degrees of freedom, of the order $O\left(10^{9}\right)$ larger than the qubit but finite. As a consequence, the changing temperature effects the coupling between qubit and calorimeter. Hence, the novelty of our model is that it describes the coupled evolution of the system and the temperature of the calorimeter (cf., [37,40,41]).

After analyzing the measurement protocol in Sec. III, we derive in Sec. IV the fluctuation relation satisfied by our system under what appears to be the natural notion of time reversal in our setup (see [8]). We then use the fluctuation relation to identify the entropy production by the driven qubit dynamics. This is the second main result of the paper.

In Sec. V, we reframe our results by deriving the form of the first and second law of thermodynamics. Finally, in Sec. VI we give a brief description of how to extend our model to a more realistic situation by taking into account the interaction, mediated by phonons, of the calorimeter with the environment.

\section{MODELING}

The system under consideration consists of a qubit with an energy gap $\hbar \omega_{0}$ that is driven by an external source and is coupled to an electron system with finite heat capacity $C$ (see Fig. 1). The total system is considered to be governed by the Schrödinger equation with a time-dependent Hamiltonian composed of three parts,

$$
H(t)=H_{2}(t)+H_{e}+H_{I}
$$

Here $H_{e}$ and $H_{I}$ denote the free Hamiltonian of the electrons and the interaction Hamiltonian between qubit and electrons. The Hamiltonians

$$
H_{2}(t)=H_{2}+H_{d}(t)
$$

acting on the qubit consist of the free Hamiltonian $H_{2}=$ $\hbar \omega_{0} a^{*} a$ and a driving Hamiltonian of the form $H_{d}(t)=$ $\lambda_{t}\left(\imath a-\imath a^{*}\right)$ varying smoothly in time. Here we write $a^{*}$ and $a$ for the creation and annihilation operators of the qubit.

During the process, we continuously measure the temperature of the electron system, henceforth referring to the electrons as a calorimeter. We assume that the number of electrons in the calorimeter is finite but very large and that the temperature is sufficiently small. Then the Sommerfeld expansion (see, e.g., Appendix $\mathrm{C}$ of [42]) may be used to evaluate the average energy of the calorimeter as a function of its temperature. Restricting to the leading-order contribution, we obtain the relation

$$
\Delta\left(T^{2}\right)=\frac{4 \mathcal{E}_{F}}{\pi^{2} k_{B}^{2} \mathcal{N}} \Delta E
$$

between the change of the squared temperature $\Delta\left(T^{2}\right)$ and the change of the average energy $\Delta E$, where $\mathcal{E}_{F}$ is the Fermi energy, $k_{B}$ is the Boltzmann constant, and $\mathcal{N}$ is the average number of electrons in the calorimeter.

We assume that the drive changes on much slower time scales than the interaction between the qubit and the calorimeter. Then the usual Born-Markov approximation (see [38,39] and cf. $[43,44]$ ) yields an effective master equation for the joint system consisting of the qubit and the temperature of the calorimeter. The details of the derivation are outlined in Appendix A. For our purposes, the master equation is conveniently written as the Itô stochastic differential equation extending the well-known stochastic Schrödinger equation (see [38,39]):

$$
\begin{aligned}
d \varphi_{t}= & \left(\frac{a_{t} \varphi_{t}}{\left\|a_{t} \varphi_{t}\right\|}-\varphi_{t}\right) d N_{t}^{+}+\left(\frac{a_{t}^{*} \varphi_{t}}{\left\|a_{t}^{*} \varphi_{t}\right\|}-\varphi_{t}\right) d N_{t}^{-} \\
& -\frac{l}{\hbar} G_{t}\left(T_{t}\right) \varphi_{t} d t+\frac{1}{2}\left[\gamma_{t}^{+}\left(T_{t}\right)\left\|a_{t} \varphi_{t}\right\|^{2}\right. \\
& \left.+\gamma_{t}^{-}\left(T_{t}\right)\left\|a_{t}^{*} \varphi_{t}\right\|^{2}\right] \varphi_{t} d t \\
d\left(T_{t}^{2}\right)= & \frac{4 \mathcal{E}_{F}}{\pi^{2} k_{B}^{2} \mathcal{N}} \hbar \omega_{t}\left(d N_{t}^{+}-d N_{t}^{-}\right)
\end{aligned}
$$

with the temperature and time-dependent non-Hermitian operator

$$
G_{t}(T)=H_{2}(t)-\imath \frac{\hbar}{2}\left[\gamma_{t}^{+}(T) a_{t}^{*} a_{t}+\gamma_{t}^{-}(T) a_{t} a_{t}^{*}\right] .
$$

In this equation, $\varphi_{t}$ and $T_{t}$ are the pure state of the qubit and the measured temperature at time $t$, respectively. The operators $a_{t}$ and $a_{t}^{*}$ denote the annihilation and creation operators in the eigenbasis of the qubit Hamiltonian $H_{2}(t)=H_{2}+H_{d}(t)$, and $\hbar \omega_{t}$ is the energy gap of $H_{2}(t)$. The processes $N_{t}^{+}$and $N_{t}^{-}$ are Poisson processes with independent increments and rates depending on $\varphi_{t}$ and $T_{t}$ via

$$
\begin{aligned}
& \left\langle d N_{t}^{+}\right\rangle=\gamma_{t}^{+}\left(T_{t}\right)\left\|a_{t} \varphi_{t}\right\|^{2} d t, \\
& \left\langle d N_{t}^{-}\right\rangle=\gamma_{t}^{-}\left(T_{t}\right)\left\|a_{t}^{*} \varphi_{t}\right\|^{2} d t .
\end{aligned}
$$

The rates $\gamma_{t}^{ \pm}(T)$ are of the form

$$
\gamma_{t}^{+}(T)=\frac{\gamma \omega_{t}}{1-e^{-\beta(T) \hbar \omega_{t}}}, \quad \gamma_{t}^{-}(T)=\frac{\gamma \omega_{t}}{e^{\beta(T) \hbar \omega_{t}}-1}
$$

for some constant $\gamma$ and the inverse temperature $\beta(T)=$ $1 / k T$. In particular, the rates satisfy the detailed balance condition $\gamma_{t}^{+}(T)=e^{\beta(T) \hbar \omega_{t}} \gamma_{t}^{-}(T)$. 


\section{MEASUREMENTS AND THEIR PROBABILITIES}

For the system described above, we study a concrete measurement protocol specified as follows: We fix a time interval $\left[t_{i}, t_{f}\right]$ and we assume that the drive vanishes at the beginning and the end of the interval, i.e., $\lambda_{t_{i}}=0=\lambda_{t_{f}}$. During the interval, we proceed as follows:

(i) At initial time $t_{i}$, we prepare the qubit randomly in one of its eigenstates $\varphi_{i}=|\uparrow\rangle$ or $\varphi_{i}=|\downarrow\rangle$. Equivalently, we assume that the qubit is directly measured in this basis.

(ii) During the time interval, we continuously measure the temperature of the calorimeter, which provides a temperature trajectory $T_{t}$.

(iii) At final time $t_{f}$, we directly measure the energy of the qubit resulting in a state $\varphi_{f}=|\uparrow\rangle$ or $\varphi_{f}=|\downarrow\rangle$.

To make the distribution of the temperature trajectory explicit, let us fix a trajectory until time $t_{i} \leqslant t \leqslant t_{f}$. We write $t_{i}=t_{0}<t_{1}<\cdots<t_{n}<t_{n+1}=t$ for the partition of the time interval such that the temperature jumps at times $t_{k}$ for $1 \leqslant k \leqslant n$ and remains constant at temperature $T_{k}$ during the interval $\left(t_{k-1}, t_{k}\right)$ for $1 \leqslant k \leqslant n+1$. Then the state of the qubit at time $t$ is given by renormalizing the vector,

$$
\begin{aligned}
\tilde{\varphi}_{t} & =U_{t, t_{n}}\left(T_{n}\right) \sqrt{\gamma_{n}} a_{n} \cdots U_{t_{2}, t_{1}}\left(T_{1}\right) \sqrt{\gamma_{1}} a_{1} U_{t_{1}, t_{0}}\left(T_{0}\right) \varphi_{i} \\
& =\left(\prod_{k=1}^{n} \gamma_{k}\right)^{1 / 2} U_{t, t_{n}}\left(T_{n}\right) \cdots a_{1} U_{t_{1}, t_{0}}\left(T_{0}\right) \varphi_{i},
\end{aligned}
$$

where for sake of brevity we set $\gamma_{k}:=\gamma_{t_{k}}^{+}\left(T_{k-1}\right), a_{k}:=a_{t}$ if $T_{k}>T_{k-1}$ and otherwise we set $\gamma_{k}:=\gamma_{t_{k}}^{-}\left(T_{k-1}\right), a_{k}:=a_{t}^{*}$. The operators $U_{t, s}(T)$ denote the two-parameter semigroup acting on the Hilbert space of the qubit given by the solution of $U_{s, s}(T)=1$ and $\frac{d}{d t} U_{t, s}(T)=-\frac{l}{\hbar} G_{t}(T) U_{t, s}(T)$ for all $s \leqslant t$. For a trajectory of the full time horizon $\left[t_{i}, t_{f}\right]$, the conditional distribution of a temperature trajectory $\left(T_{t}\right)_{t}$ and a final measurement result $\varphi_{f}$ is hence given by

$$
\begin{aligned}
\mathbb{P}\left(T_{t}, \varphi_{f} \mid \varphi_{i}\right): & =\left|\left\langle\varphi_{f} \mid \tilde{\varphi}_{t_{f}}\right\rangle\right|^{2} \\
& =\left(\prod_{k=1}^{n} \gamma_{k}\right)\left|\left\langle\varphi_{f}\left|U_{t_{f}, t_{n}}\left(T_{n}\right) \cdots U_{t_{1}, t_{0}}\left(T_{0}\right)\right| \varphi_{i}\right\rangle\right|^{2} .
\end{aligned}
$$

\section{FLUCTUATION RELATION AND ENTROPY PRODUCTION}

To derive a fluctuation relation for the investigated process, we choose a natural time reversal. For the sake of distinction, we henceforth refer to the process described in Sec. II, Eq. (2), as the forward process. The dynamics of the reversed process is also determined by Eq. (2), where we replace $G_{t}(T)$ by the operator $G_{t}^{R}(T)$ given by

$$
G_{t_{f}-t}^{R}(T)=-H_{2}(t)-\imath \frac{\hbar}{2}\left[\gamma^{+}(T)^{*} a_{t}^{*} a_{t}+\gamma_{t}^{-}(T) a_{t} a_{t}^{*}\right] .
$$

The resulting equation may equivalently be derived by inverting the initial Schrödinger equation of the combined quantum system consisting of a qubit and a calorimeter and performing the same approximation described in Sec. II. We apply the same protocol to the time-reversed process, that is, at time $t_{i}$ we prepare the qubit, we observe the temperature during the time interval $\left[t_{i}, t_{f}\right]$, and we measure the qubit directly at final time $t_{f}$. Proceeding as in Sec. III, we may analogously derive the distribution on the path space for the reversed process.

We now compare a trajectory of the forward process, given by the initial and final qubit state, $\varphi_{i}$ and $\varphi_{f}$, and the temperature $T_{t}$, with the trajectory of the reversed process that runs backward in time, i.e., the trajectory that starts with a qubit in state $\varphi_{f}$, yields the temperatures $T_{t_{f}-t}$, and results in $\varphi_{i}$ in the final measurement.

To compare the path distribution of the forward process $\mathbb{P}$ and the reversed process $\mathbb{P}^{R}$, we may observe the following two facts. First, for each jump in a trajectory of the forward process there is a jump in the opposite direction in the corresponding trajectory of the reversed process. However, the jumping rates are different. Second, the evolution in between temperature jumps is governed by the two-parameter semigroups $U_{t, s}(T)$ with generator $-\frac{l}{\hbar} G_{t}(T)$ for the forward process and by $U_{t, s}^{R}(T)$ with generator $-\frac{l}{\hbar} G_{t}^{R}(T)$ for the reversed process, respectively. Since $G_{t_{f}-t}^{R}(T)=-G_{t}(T)^{*}$, the associated semigroups satisfy

$$
U_{t, s}^{R}(T)=U_{t_{f}-s, t_{f}-t}(T)^{*}
$$

for all $s \leqslant t$. We may now compare the expressions (4) and (5) for the density of the forward process with the corresponding expressions for density of the backward process. Gathering the coefficients then immediately yields

$$
\mathbb{P}\left(T_{t}, \varphi_{f} \mid \varphi_{i}\right)=e^{J} \mathbb{P}^{R}\left(T_{t_{f}-t}, \varphi_{i} \mid \varphi_{f}\right) .
$$

Here $J$ is the function of the path of the forward process given by $J:=\sum_{k=1}^{n} J_{k}$ with a contribution

$$
J_{k}:=\left[\ln \gamma_{t_{k}}^{x_{k}}\left(T_{k-1}\right)-\ln \gamma_{t_{k}}^{-x_{k}}\left(T_{k}\right)\right]
$$

for each jump. Here, as before, $T_{i}=T_{0}, T_{1}, \ldots, T_{n}=T_{f}$ is the observed temperature trajectory, $t_{i}<t_{1}<\cdots t_{n}<t_{f}$ are the jumping times, and $x_{1}, \ldots, x_{n} \in\{+1,-1\}$ are the jumping directions $x_{k}:=d N_{t_{k}}^{+}-d N_{t_{k}}^{-}$. Using the particular form of the rates, we may conveniently write $J$ in the symmetric form

$$
J_{k}=\bar{\beta}_{k} \hbar \omega_{k} x_{k}+\ln \sinh \left(\frac{\beta_{k} \hbar \omega_{k}}{2}\right)-\ln \sinh \left(\frac{\beta_{k-1} \hbar \omega_{k}}{2}\right),
$$

where for simplicity $\omega_{k}:=\omega_{t_{k}}, \beta_{k}:=1 / k_{B} T_{k}$, and $\bar{\beta}_{k}:=$ $\left(\beta_{k-1}+\beta_{k}\right) / 2$. Since Eq. (2) is derived in the regime of low temperature neglecting higher orders, i.e., the Sommerfeld expansion, the difference $\left(\beta_{k}-\beta_{k-1}\right) \hbar \omega$ is negligible and we obtain $J_{k}=\bar{\beta}_{k} \hbar \omega_{k} x_{k}$ within the approximation. Using Eq. (1), we find that the entropy flux coincides with the change of the equilibrium entropy of the fermionic calorimeter $S_{\text {eq }}(T)=\frac{\pi^{2} k_{B} \mathcal{N}}{2 \mathcal{E}_{F}} T$ in the Sommerfeld approximation (see [45], p. 48):

$$
J_{k}=\frac{\omega_{k} x_{k}}{\bar{T}}=S_{\mathrm{eq}}\left(T_{k}\right)-S_{\mathrm{eq}}\left(T_{k-1}\right)
$$

with $\bar{T}:=\left(T_{k-1}+T_{k}\right) / 2$ satisfying within the approximation $\hbar \omega_{k} / \bar{T}=\bar{\beta} \hbar \omega$.

The entropy flux $J$ is the dynamical contribution to the total entropy production. Another contribution is given by the entropy of the initial preparation and the final measurement of the qubit given by $S_{i / f}(\varphi)=-\ln \mathbb{P}_{i / f}(\varphi)$, where $\mathbb{P}_{i / f}(\varphi)$ is the probability that the state is initially prepared in the state 
$\varphi \in\{\uparrow, \downarrow\}$ or, respectively, finally observed in $\varphi$. The entropy production of the qubit along a trajectory is then given by

$$
\sigma:=S_{f}-S_{i}+J .
$$

Equation (6) immediately implies $\left\langle e^{-\sigma}\right\rangle=1$ for the expectation value with respect to the forward process. In particular, it follows that $\langle\sigma\rangle \geqslant 0$.

The derived entropy production can be compared with the approaches to entropy production by Horowitz and Parrondo [34] and by Breuer [46] in the limit of an infinite calorimeter, i.e., when the heat capacity of the calorimeter becomes infinite. In this case, the resulting temperature change is negligible during the driving period, and the work and heat measurement strategy needs to be reconsidered. Moreover, in [46] the drive is considered as a small perturbation of the free evolution in the sense that the time dependence of the energy gap of the qubit Hamiltonian is neglected. In this case, the entropy flux takes the form

$$
J=-\sum_{k=1}^{n} \beta \hbar \omega_{0} x_{k}=-\beta \hbar \omega_{0}\left(N_{t_{f}}^{+}-N_{t_{f}}^{-}\right),
$$

where $\beta=1 / k T$ denotes the inverse temperature and where we assume without loss of generality that the Poisson processes start at $N_{0}^{+}=N_{0}^{-}=0$. Taking the expectation value, we recover the entropy flux of $[34,46]$. Due to the final measurement of the qubit, the expectation of the entropy production $\langle\sigma\rangle$ is in general larger than or equal to the entropy production in [46].

\section{FIRST AND SECOND LAW OF THERMODYNAMICS}

For the first law, we concentrate on the qubit. Suppose that at time $t$, the state of the qubit is given by $\varphi_{t}$ and the calorimeter has temperature $T$. Then the expectation value of the energy of the qubit is given by

$$
\mathcal{E}(t):=\left\langle H_{2}(t)\right\rangle \varphi_{t}
$$

with the qubit Hamiltonian $H_{2}(t)=H_{2}+H_{d}(t)$. Following $[47,48]$, the work per unit of time performed on the qubit at time $t$ is given by

$$
\mathcal{W}(t):=\langle *\rangle * \frac{d}{d t} H_{2}(t) \varphi_{t}=\langle *\rangle * \frac{d}{d t} H_{d}(t) \varphi_{t} .
$$

Furthermore, the heat per unit of time emitted by the qubit into the calorimeter at time $t$ is given by

$$
d \mathcal{Q}(t):=\hbar \omega_{t}\left(d N_{t}^{+}-d N_{t}^{-}\right) .
$$

Indeed, a straightforward computation with (2) yields the stochastic form of the first law of thermodynamics in expectation value:

$$
\langle d \mathcal{E}(t)\rangle_{\varphi_{t}}=\mathcal{W}_{t} d t-\langle d \mathcal{Q}(t)\rangle_{\varphi_{t}},
$$

where $\langle\cdot\rangle_{\varphi_{t}}$ denotes the expectation value conditioned on the qubit being in state $\varphi_{t}$.

The second law of thermodynamics may be derived in the limit of an infinite heat capacity. In this case, the calorimeter forms an infinite heat bath for the qubit with constant temperature $T$. The entropy flux $J$ then simplifies to $J=-\beta Q$, where for each trajectory, $Q:=\sum_{k=1}^{n} Q_{k}$ is the total heat emitted by the qubit. The total entropy change in the qubit along a trajectory is hence given by

$$
\Delta S:=S_{f}-S_{i}=\sigma+\beta Q .
$$

Since $\langle\sigma\rangle \geqslant 0$, taking the expectation value yields the familiar form of the second law of thermodynamics,

$$
\langle\Delta S\rangle \geqslant \beta\langle Q\rangle \text {. }
$$

\section{INCLUDING A PHONON BATH}

The model studied so far can be easily extended to the case in which the calorimeter emerges into a phonon heat bath. We assume that the interaction between the calorimeter and the phonon bath is weaker but much faster than the interaction between the the qubit and the calorimeter. Then the energy exchange between the calorimeter and phonons only depends on the respective temperatures. For a calorimeter made of ordinary metal, for instance, we have

$$
\dot{Q}_{e p}=\Sigma_{e p}\left(T^{5}-T_{p}^{5}\right),
$$

where $T$ is the temperature of the electron calorimeter, $T_{p}$ is the temperature of the phonon bath, $Q_{e p}$ is the heat emitted by the calorimeter into the phonon bath, and $\Sigma_{e p}$ is a material-dependent constant (see [49]). For materials with a more sophisticated structure, e.g., thin metallic layers, the law (7) may be different (see [50]). For simplicity, we assume that the energy exchange is deterministic. Using, as before, the approximation provided by the Sommerfeld expansion to relate energy changes and temperature changes in the calorimeter via Eq. (1), we find that the second equation of (2) has to be replaced by

$$
d\left(T_{t}^{2}\right)=\frac{4 \mathcal{E}_{F}}{\pi^{2} k_{B}^{2} \mathcal{N}}\left[\hbar \omega_{t}\left(d N_{t}^{+}-d N_{t}^{-}\right)+\Sigma_{e p}\left(T_{p}^{5}-T_{t}^{5}\right) d t\right] .
$$

\section{CONCLUSIONS}

Summarizing, we have established a mathematical model for the dynamics of an externally driven qubit interacting with a continuously monitored, finite-size calorimeter. The resulting quantum stochastic evolution (2) extends the usual stochastic Schrödinger equation by incorporating the changing temperature of the calorimeter and its backaction on the measurement process. We derived an explicit expression for the entropy production, and we demonstrated a corresponding fluctuation relation. As a first application, we verified the first law of thermodynamics and proved the second law in reasonably limiting cases. Finally, we indicated how the model can be extended easily to comprise a common phonon bath.

The model described here is intended as a first approach to the dynamics offering a simple presentation. In particular, we treated only leading terms in the Sommerfeld expansion. It might be interesting also to analyze higher-order terms in order to connect to current developments that suggest a microcanonical treatment of the calorimeter [41]. Furthermore, experiments suggest that a more realistic model should treat the phonon bath stochastically as well. Such a model is currently being investigated numerically [51] in order to analyze the fluctuating thermodynamic quantities beyond the fluctuation relation. In some cases, an analytic treatment seems feasible, 
too. Currently designed experiments address the fluctuation relation derived here. In this way, the implications of the finite size of the calorimeter are tested (cf. [52]) and the non-Markovianity of the dynamics can be quantified.

\section{ACKNOWLEDGMENTS}

The authors would like to thank Brecht Donvil, Raphaël Chétrite, Yuri Galperin, Ronnie Kosloff, and Samu Suomela for fruitful discussions and comments. This work has been supported by the Academy of Finland (Projects No. 272218, No. 284594, and No. 271983).

\section{APPENDIX A: SEPARATING TIME SCALES}

The fastest time scale in the interaction between qubit and calorimeter is the electron-electron interaction in the calorimeter. This interaction is much faster than any other time scale involved, and it does not effect the average energy. Therefore, we treat the self-interaction as an average effect in the van-Hove weak-coupling limit [53-55]. On the relevant time scales, the calorimeter then can be assumed to be always in a well-defined temperature state characterized by its mean energy.

The second fastest time scale is the interaction between the qubit and the calorimeter. The drive changes on a much slower time scale. Then the qubit-calorimeter interaction can be treated by the usual Born-Markov approximation (see [38], Secs. 3.3 and 8.4, or [39], Sec. 3.2), where the drive is considered to be constant during the interaction. Since we only study energy changes in the calorimeter, we may for this purpose regard the calorimeter as free fermions with a corresponding free Hamiltonian $H_{e}=\sum_{k} \epsilon_{k} c_{k}^{*} c_{k}$, and we may take the interaction Hamiltonian of the form

$$
H_{I}=\sum_{k \neq \ell} g_{k, \ell}\left(a+a^{*}\right) c_{k}^{*} c_{\ell},
$$

where the sums are taken over all (pairs of) fermionic modes with corresponding energy $\epsilon_{k}$ and with some interaction energies $g_{k, \ell}=\overline{g_{\ell, k}}$.

\section{APPENDIX B: PURIFICATION OF THE TEMPERATURE STATE}

To simplify the further derivation, it is convenient to pass from the temperature state of inverse temperature $\beta$ to a pure state. For the temperature state, this is achieved by considering a new set of fermionic annihilation operators $\tilde{c}_{k}, \tilde{h}_{\ell}$ satisfying

$$
c_{k}=\sqrt{1-f_{k}} \tilde{c}_{k}+\sqrt{f_{k}} \tilde{h}_{k}^{*}
$$

where $f_{k}:=1 /\left(e^{\beta\left(\epsilon_{k}-\mu\right)}+1\right)$ denotes the Fermi function for inverse temperature $\beta$ and chemical potential $\mu$. That is, the $c_{k}$ 's can be obtained from $\tilde{c}_{k}$ and $\tilde{h}_{\ell}$ by a suitable Bogoliubov transform, which then also provides the fermionic annihilation operators

$$
h_{\ell}:=\sqrt{1-f_{\ell}} \tilde{h}_{\ell}-\sqrt{f_{\ell}} \tilde{c}_{\ell}^{*},
$$

anticommuting with the $c_{k}$ 's.

\section{APPENDIX C: INTERACTION PICTURE}

It is common to assign the negative energy $\epsilon_{k}$ to the fermionic mode corresponding to $h_{k}$. That is, we consider the free Hamiltonian $\tilde{H}_{e}=\sum_{k} \epsilon_{k}\left(c_{k}^{*} c_{k}-h_{k}^{*} h_{k}\right)$ and we proceed to the interaction picture with respect to $\tilde{H}_{e}$.

For the qubit, it is expedient to write the drive in the form

$$
H_{d}(t)=\frac{1}{2} \hbar \omega_{0} \tan \theta_{t}\left(\iota a-\imath a^{*}\right)
$$

with some smooth parameter $-\pi / 2<\theta_{t}<\pi / 2$. For this choice of parameters, the total Hamiltonian of the qubit, $H_{2}(t)=H_{2}+H_{d}(t)$, is diagonalized by the unitary operator

$$
U_{t}:=\left(\begin{array}{cc}
\cos \left(\theta_{t} / 2\right) & -\imath \sin \left(\theta_{t} / 2\right) \\
-l \sin \left(\theta_{t} / 2\right) & \cos \left(\theta_{t} / 2\right)
\end{array}\right),
$$

with the generator $H_{A}(t):={ }_{\imath} \dot{U}_{t} U_{t}^{*}=-\frac{1}{2} \dot{\theta}_{t}\left(a+a^{*}\right)$. For the derivation of the master equation, we treat the drive adiabatically, i.e., $H_{d}(t)$ is treated as constant on the time scale of the interaction of qubit and calorimeter. For the derivation of the master equation, we then pass to the interaction picture with respect to the unitary transform $U_{t}$.

It is worth noting that the field operator $a+a^{*}$ commutes with $H_{A}(t)$ and hence satisfies $U_{t}\left(a+a^{*}\right) U_{t}^{*}=a+a^{*}$. Proceeding to the interaction picture, therefore, does not effect the interaction Hamiltonian $H_{I}$. However, it is convenient to write $H_{I}$ in terms of the eigenbasis of the qubit Hamiltonian $H_{2}+H_{d}(t)$. We denote by $\tilde{a}$ the corresponding annihilation operator, which then satisfies $a=\tilde{a}+\frac{l}{2} \sin \theta\left(1-2 \tilde{a}^{*} \tilde{a}\right)$. Using $g_{k, \ell}=\overline{g_{\ell, k}}$, we obtain for the interaction Hamiltonian (in the interaction picture)

$$
\begin{aligned}
\tilde{H}_{I}= & \sum_{k \neq \ell} g_{k, \ell} e^{l\left(\epsilon_{k}-\epsilon_{\ell}\right) t}\left(e^{\imath \hbar \tilde{\omega} t} \tilde{a}^{*}+e^{-i \hbar \tilde{\omega} t} \tilde{a}\right) \\
& \times\left(\sqrt{1-f_{k}} \sqrt{1-f_{\ell}} \tilde{c}_{k} \tilde{c}_{\ell}+\sqrt{f_{k}} \sqrt{f_{\ell}} \tilde{h}_{k}^{*} \tilde{h}_{\ell}\right. \\
& \left.+\sqrt{1-f_{k}} \tilde{f}_{\ell} \tilde{c}_{k} \tilde{h}_{\ell}^{*}+\sqrt{f_{k}} \sqrt{1-f_{\ell}} \tilde{h}_{k} \tilde{c}_{\ell}\right),
\end{aligned}
$$

where $\hbar \tilde{\omega}=\hbar \omega_{0} / \cos \theta$ is the spectral gap of the qubit Hamiltonian $\mathrm{H}_{2}+\mathrm{H}_{d}(t)$. From this point on, the derivation of the master equation proceeds according to the standard textbook Born-Markov and rotating-wave approximations. We refer the reader to, e.g., [38] (Sec. 6), or to [37,52], where similar models are treated.
[1] R. Landauer, IBM J. Res. Dev. 5, 183 (1961).

[2] J. D. Bekenstein, Phys. Rev. Lett. 46, 623 (1981).

[3] S. Lloyd, Nature (London) 406, 1047 (2000).

[4] H. S. Leff and A. F. Rex, Maxwell's Demon 2: Entropy, Classical and Quantum Information, Computing, 2nd ed. (Institute of Physics, Bristol, UK, 2003), p. 485.
[5] D. R. Schmidt, C. S. Yung, and A. N. Cleland, Appl. Phys. Lett. 83, 1002 (2003).

[6] S. Gasparinetti, K. L. Viisanen, O.-P. Saira, T. Faivre, M. Arzeo, M. Meschke, and J. P. Pekola, Phys. Rev. Appl. 3, 014007 (2015).

[7] F. Giazotto, T. T. Heikkilä, A. Luukanen, A. M. Savin, and J. P. Pekola, Rev. Mod. Phys. 78, 217 (2006). 
[8] J. P. Pekola, P. Solinas, A. Shnirman, and D. V. Averin, New J. Phys. 15, 115006 (2013).

[9] J. Clarke and F. K. Wilhelm, Nature (London) 453, 1031 (2008).

[10] D. V. Averin, A. B. Zorin, and K. K. Likharev, Zh. Eksp. Teor. Fiz. 88, 692 (1984) [J. Exp. Theor. Phys. 61, 407 (1984)].

[11] M. Büttiker, Phys. Rev. B 36, 3548 (1987).

[12] Y. Makhlin, G. Schön, and A. Shnirman, Nature (London) 398, 305 (1999).

[13] J. Koch, T. M. Yu, J. Gambetta, A. A. Houck, D. I. Schuster, J. Majer, A. Blais, M. H. Devoret, S. M. Girvin, and R. J. Schoelkopf, Phys. Rev. A 76, 042319 (2007).

[14] J. A. Schreier, A. A. Houck, J. Koch, D. I. Schuster, B. R. Johnson, J. M. Chow, J. M. Gambetta, J. Majer, L. Frunzio, M. H. Devoret, S. M. Girvin, and R. J. Schoelkopf, Phys. Rev. B 77, 180502(R) (2008).

[15] V. Bouchiat, D. Vion, P. Joyez, D. Esteve, and M. H. Devoret, Phys. Scr. T76, 165 (1998).

[16] Y. Nakamura, Y. A. Pashkin, and J.-S. Tsai, Nature (London) 398, 786 (1999).

[17] Y. Nakamura, Nature (London) 459, 516 (2009).

[18] D. J. Evans and D. J. Searles, Phys. Rev. E 50, 1645 (1994).

[19] G. Gallavotti and E. G. D. Cohen, Phys. Rev. Lett. 74, 2694 (1995).

[20] C. Jarzynski, Phys. Rev. Lett. 78, 2690 (1997).

[21] G. E. Crooks, J. Stat. Phys. 90, 1481 (1997).

[22] J. Kurchan, J. Phys. A 31, 3719 (1998).

[23] J. L. Lebowitz and H. Spohn, J. Stat. Phys. 95, 333 (1999).

[24] D.-Q. Jiang, M. Qian, and M.-P. Qian, Mathematical Theory of Nonequilibrium Steady States, Lecture Notes in Mathematics Vol. 1833 (Springer, Berlin, Heidelberg, 2004), p. 276.

[25] R. Chétrite and K. Gawędzki, Commun. Math. Phys. 282, 469 (2008).

[26] K. Sekimoto, Stochastic Energetics, Lecture Notes in Physics Vol. 799 (Springer, Berlin, Heidelberg, 2010), p. 322.

[27] J. Kurchan, arXiv:cond-mat/0007360 (cond-mat.stat-mech).

[28] S. Mukamel, Phys. Rev. Lett. 90, 170604 (2003).

[29] W. De Roeck and C. Maes, Phys. Rev. E 69, 026115 (2004).

[30] C. Jarzynski and D. K. Wójcik, Phys. Rev. Lett. 92, 230602 (2004).

[31] S. Attal and K. Gawędzki (unpublished).
[32] M. Campisi, P. Hänggi, and P. Talkner, Rev. Mod. Phys. 83, 771 (2011).

[33] R. Chétrite and K. Mallick, J. Stat. Phys. 148, 480 (2012).

[34] J. M. Horowitz and J. M. R. Parrondo, New J. Phys. 15, 085028 (2013).

[35] T. Albash, D. A. Lidar, M. Marvian, and P. Zanardi, Phys. Rev. E 88, 032146 (2013).

[36] P. Solinas, D. V. Averin, and J. P. Pekola, Phys. Rev. B 87, 060508(R) (2013).

[37] J. P. Pekola, S. Suomela, and Y. M. Galperin, J. Low Temp. Phys. 184, 1015 (2016).

[38] H.-P. Breuer and F. Petruccione, The Theory of Open Quantum Systems, reprint ed. (Oxford University Press, Oxford, 2002), p. 636.

[39] H. M. Wiseman and G. J. Milburn, Quantum Measurement and Control, 1st ed. (Cambridge University Press, Cambridge, 2009), p. 478.

[40] T. L. van den Berg, F. Brange, and P. Samuelsson, New J. Phys. 17, 075012 (2015).

[41] S. Suomela, A. Kutvonen, and T. Ala-Nissila, Phys. Rev. E 93, 062106 (2016).

[42] N. W. Ashcroft and D. Mermin, Solid State Physics, 1st ed. (Saunders College Publishing, Philadelphia, 1976), p. 848.

[43] H.-P. Breuer, Phys. Rev. A 75, 022103 (2007).

[44] C. Pellegrini, J. Stat. Phys. 154, 838 (2014).

[45] A. L. Fetter and J. D. Walecka, Quantum Theory of ManyParticle Systems, 1st ed., Pure \& Applied Physics (McGraw-Hill College, San Fransisco, 1971), pp. XIV and 601.

[46] H.-P. Breuer, Phys. Rev. A 68, 032105 (2003).

[47] W. Pusz and S. L. Woronowicz, Commun. Math. Phys. 58, 273 (1978).

[48] R. Alicki, J. Phys. A 12, L103 (1979).

[49] F. C. Wellstood, C. Urbina, and J. Clarke, Phys. Rev. B 49, 5942 (1994).

[50] S. Cojocaru and D.-V. Anghel, Phys. Rev. B 93, 115405 (2016).

[51] B. Donvil, Master's thesis, KU Leuven, 2016.

[52] S. Suomela, P. Solinas, J. P. Pekola, J. Ankerhold, and T. AlaNissila, Phys. Rev. B 90, 094304 (2014).

[53] L. van Hove, Physica 21, 517 (1954).

[54] E. B. Davies, Commun. Math. Phys. 39, 91 (1974).

[55] E. B. Davies, Quantum Theory of Open Systems (Academic Press, London, 1976), p. 171. 